\title{
A leptospirose humana como doença duplamente negligenciada no Brasil
}

\author{
Human leptospirosis as a doubly neglected disease in Brazil
}

Mário Henrique da Mata Martins (https://orcid.org/0000-0002-1370-300X) ${ }^{1}$

Mary Jane Paris Spink (https://orcid.org/0000-0003-1672-505X) ${ }^{2}$
${ }^{1}$ Fundação Getúlio Vargas. Av. 9 de Julho 2029, Edifício John F. Kennedy, Bela Vista. 01313-902 São Paulo SP Brasil. martins.mariodamata@ gmail.com

${ }^{2}$ Pontifícia Universidade Católica de São Paulo. São Paulo SP Brasil.

\begin{abstract}
The aim of this article is to analyze discrepancies and informational gaps which produce a doubly neglected version of human leptospirosis in the Brazilian public health policy. To achieve this goal, we compared data on morbidity, mortality, hospital and social costs, population profiles, vector habits, social health determinants and diagnostic practices related to leptospirosis with another disease of higher recognition in Brazil: dengue fever. Our analysis shows that the arbitrariness of criteria for assigning health priorities, the invisibility of the population profile of human leptospirosis in official data and its mimetic character in clinic corroborate the production of a version of human leptospirosis that is invisible and, because of that, doubly neglected by the Brazilian public health policy. We conclude that these discrepancies and informational gaps are related to the fact that human leptospirosis affects a population which the State has no interest in keeping alive.
\end{abstract}

Key words Leptospirosis, Neglected diseases, $\mathrm{Pu}$ blic health policies
Resumo O objetivo do presente artigo é analisar as discrepâncias e lacunas informacionais que produzem uma versão duplamente negligenciada da leptospirose humana na política pública de saúde brasileira. Para alcançar esse objetivo, comparamos dados de morbidade, mortalidade, custos hospitalares e sociais, perfis populacionais, hábitos dos vetores, determinantes sociais de saúde e práticas diagnósticas relacionadas à leptospirose com outra doença de maior reconhecimento no país: a dengue. Nossa análise mostra que a arbitrariedade dos critérios para atribuições de prioridades de intervenção em saúde, a invisibilidade do perfil populacional da leptospirose humana nos dados oficiais da politica de saúde e seu caráter clínico mimético corroboram para a produção de uma versão da leptospirose humana que é invisivel e, portanto, duplamente negligenciada pela política pública de saúde brasileira. Concluímos que essas discrepâncias e lacunas informacionais relacionam-se ao fato de que a leptospirose humana acomete uma população que o Estado não tem interesse em manter viva.

Palavras-chave Leptospirose, Doenças negligenciadas, Políticas Públicas de Saúde 


\section{Introdução}

O objetivo do presente artigo é analisar as discrepâncias e lacunas informacionais que produzem uma versão duplamente negligenciada da leptospirose humana na política pública de saúde brasileira. Nossa meta é promover um debate sobre a invisibilidade do impacto social e econômico dessa enfermidade e do perfil das pessoas acometidas pela doença de forma a propiciar meios de fomentar seu reconhecimento e enfrentamento como uma doença negligenciada no Brasil.

A patogênese da leptospirose humana ainda é pouco conhecida ${ }^{1}$, sua transmissão para seres humanos ocorre de modo acidental por intermédio da urina de animais contaminados por uma bactéria ${ }^{2}$ e, embora tenha sido caracterizada historicamente como uma doença rural, atinge hoje cada vez mais as populações urbanas ${ }^{3,4}$. Essa enfermidade está relacionada tanto a condições comportamentais ${ }^{5}$ como socioambientais ${ }^{6}$ e é recorrente em áreas pobres, com altos índices de desigualdade social, de países em desenvolvimento, causando grandes perdas sociais e econômicas ${ }^{7,8}$.

Embora seja potencialmente letal, seu impacto na saúde da população ainda é subestimado9. No plano político e midiático a doença tem pouca ou nenhuma visibilidade, o que a torna marginalizada e desconhecida pelo público geral ${ }^{10,11}$. Em virtude de sua estreita relação com a pobreza, o desinteresse público por sua resolução e a eventual necessidade de custosos tratamentos permanentes ou de longa duração após a infecção, foi classificada na literatura internacional como uma Doença Tropical Negligenciada (DTN), classificação referente a doenças prevalentes em populações de áreas pobres, que não detém condições econômicas e de infraestrutura para mobilizar o investimento nas enfermidades de que convalescem e não despertam o interesse de grandes indústrias farmacêuticas ou mesmo de seus governantes para a produção de medicamentos e vacinas $^{7,8}$.

Nesse ensejo, o Brasil possui uma porcentagem alta de DTN como a esquistossomose, hanseníase, tracoma, leptospirose (quase 90\% dos casos), dengue, malária, ambas as formas de leishmaniose e possivelmente doença de Chagas, o que se relaciona de forma direta às desigualdades socioeconômicas do país ${ }^{8}$. De modo a fazer frente a essa problemática, o país vem investindo em editais para essas doenças pelo Programa de Pesquisa e Desenvolvimento em Doenças Negligenciadas, que tem por objetivo promover a inovação através do desenvolvimento de medi- camentos para os programas públicos de saúde. Por meio desse programa, já foram lançados dois editais temáticos que financiaram 140 projetos com um investimento total de $\mathrm{R} \$ 39$ milhões, além de editais lançados para doenças específicas. O Quadro 1 apresenta os editais lançados até 2010 que contemplam as DTN e seus respectivos investimentos.

Apesar do reconhecimento internacional da leptospirose como uma DTN, os editais nacionais não fazem qualquer menção a investimentos de pesquisa e intervenção para esse agravo. Isso porque foram definidas, por meio de dados epidemiológicos, demográficos e de impacto, sete prioridades de atuação que compõem o programa em doenças negligenciadas no Brasil: dengue, doença de Chagas, leishmaniose, hanseníase, malária, esquistossomose e tuberculose. Em termos de investimento governamental direto, a leptospirose humana sequer é considerada uma doença negligenciada no país e não foi contemplada com um edital conjunto ou específico ${ }^{12}$.

Com base nessa ausência, propusemos discutir neste artigo a dupla negligência da leptospirose humana na rede de saúde, uma negligência que se relaciona a suas características e impactos característicos de uma doença negligenciada e sua falta de reconhecimento como tal pela política de saúde brasileira. Para alcançar esse objetivo, realizamos um comparativo de informações com uma DTN que recebe muito mais atenção governamental: a dengue. Não temos a pretensão de oferecer resultados e provas de correlação entre as duas doenças e muito menos afirmar que não se deve investir na dengue, mas explicitar os mecanismos por meio dos quais uma doença se torna DTN e, logo, um problema de saúde pública reconhecido, enquanto a outra não. A partir disso, discutimos os pressupostos biopolíticos que fundamentam essa segregação e levam o Estado a privilegiar um grupo populacional em detrimento de outro.

\section{Métodos e materiais}

O presente estudo fez uso de três estratégias metodológicas pautadas na análise de múltiplas fontes de pesquisa ${ }^{13}$ : uma análise comparativa dos dados de morbidade, mortalidade e custos financeiros e sociais da dengue e da leptospirose humana na rede de saúde, seguida de uma análise comparativa das características populacionais e dos determinantes sociais de ambas as enfermidades e, por fim, um estudo de caso sobre ca- 
Quadro 1. Grandes editais temáticos na área de doenças negligenciadas no Brasil.

\begin{tabular}{|c|l|l|}
\hline \multicolumn{1}{|c|}{ Ano } & \multicolumn{1}{|c|}{ Edital } & \multicolumn{1}{c|}{ Recursos } \\
\hline 2003 & Rede Tuberculose & $\mathrm{R} \$ 1,9$ milhões \\
\hline 2004 & Dengue & $\mathrm{R} \$ 945$ mil \\
\hline 2005 & Hanseníase & $\mathrm{R} \$ 2,5$ milhões \\
\hline 2006 & Doenças Negligenciadas & $\mathrm{R} \$ 17,0$ milhões \\
\hline 2008 & Doenças Negligenciadas & $\mathrm{R} \$ 22,0$ milhões \\
\hline 2009 & Rede Malária & $\mathrm{R} \$ 15,4$ milhões \\
\hline 2009 & Rede Dengue & $\mathrm{R} \$ 22,7$ milhões \\
\hline
\end{tabular}

Fonte: Adaptado de Ministério da Saúde ${ }^{12}$.

sos de dengue e leptospirose humana na rede de saúde.

De modo a cumprir com a primeira estratégia metodológica desse trabalho, fizemos uso das informações dispostas no Sistema de Informação sobre Agravos de Notificação (Sinan) e no Sistema de Informações Hospitalares do SUS (SIH/SUS) sobre a dengue e a leptospirose humana. Além disso, comparamos três indicadores de impacto social dessas enfermidades: Anos Potenciais de Vida Perdidos (AVP), que quantifica o número de anos não vividos em virtude de óbito em idade na qual se considera a morte prematura; Anos de Trabalho Perdidos (ATP), que quantifica a perda de tempo de trabalho em virtude de óbito antes ou durante a faixa etária produtiva; e impacto salarial de cada enfermidade, referente às perdas salariais por falta no trabalho. Com base nessas informações, discutimos a aparente arbitrariedade dos critérios de atribuição de prioridades de intervenção em saúde.

Em seguida, para cumprir a segunda estratégia metodológica deste trabalho, discutimos as diferenças e semelhanças entre os perfis populacionais de ambas as enfermidades. Para isso, selecionamos na base de dados do Sinan quatro variáveis relacionadas à leptospirose (2007-2015) e à dengue (2007-2012) nos períodos recentes disponíveis na plataforma. Essas quatro variáveis foram idade, sexo, cor/raça e escolaridade. Em seguida identificamos as principais lacunas de informação sobre esses perfis populacionais e buscamos complementá-las por meio da associação com informações relativas ao comportamento dos vetores e aos determinantes sociais de ambas as enfermidades. Com base nessas informações, discutimos uma invisibilidade populacional da leptospirose humana pautada na produção de lacunas informacionais que obliteram grupos marginalizados.
Por fim, de forma a cumprirmos a última estratégia metodológica, buscamos compreender como se produz uma lacuna nas informações sobre a leptospirose humana na prática de saúde por meio de um estudo de tipo etnográfico no qual acompanhamos a recepção, diagnóstico e tratamento de casos de pessoas que tiveram leptospirose em um hospital especializado em doenças infecciosas, com destaque a um estudo de caso sobre um usuário que teve dengue e leptospirose ao mesmo tempo. Comparamos as informações obtidas com os dados previamente recuperados do Sinan sobre o impacto de ambas as doenças na rede de saúde e discutimos a produção de uma invisibilidade pautada no caráter mimético da leptospirose e na variância de atenção da rede de saúde para a doença.

Em virtude desse último procedimento, a pesquisa foi submetida ao comitê de ética da Pontifícia Universidade Católica de São Paulo (PUC) e foi aprovada.

\section{Resultados e discussão}

\section{Quais os critérios disponíveis para priorizar uma doença?}

Neste tópico, discutimos os critérios para considerar uma doença, a dengue, como um problema de saúde pública enquanto outra, a leptospirose, se torna duplamente negligenciada. Na Tabela 1 estão listados os valores absolutos de casos confirmados e óbitos por leptospirose humana e dengue entre os anos 2000 e 2015. É possível notar que a dengue apresenta um registro de casos 174 vezes maior do que a leptospirose no território nacional. Vista a coluna de casos, não parece nada questionável o investimento maciço feito nessa enfermidade. Todavia, se analisarmos a outra dupla de colunas, constatamos uma situação contraditória: o número de óbitos por leptospirose humana é três vezes maior do que o de óbitos por dengue. Nesse caso, podemos pensar em dois critérios para priorizar um agravo na política pública: um critério de morbidade e outro de mortalidade.

Todavia, esses critérios só fazem sentido se comparados aos custos dessas enfermidades, tendo em vista que elas impactam de maneiras diferentes os cofres públicos. Para este critério financeiro propusemos analisar os custos hospitalares e os custos sociais de ambas as doenças.

Em relação ao custo hospitalar, o investimento total para dengue no Brasil nos últi- 
Tabela 1. Casos e óbitos confirmados por leptospirose e por dengue no Brasil (2000-2015*).

\begin{tabular}{|c|c|c|c|c|}
\hline \multirow{2}{*}{ Ano } & \multicolumn{2}{|c|}{ Casos $^{\star}$} & \multicolumn{2}{|c|}{ Óbitos ${ }^{\star}$} \\
\hline & Dengue & Leptospirose & Dengue & Leptospirose \\
\hline 2000 & 135.228 & 4.208 & 3 & 351 \\
\hline 2001 & 385.783 & 3.708 & 41 & 436 \\
\hline 2002 & 696.472 & 2.796 & 121 & 332 \\
\hline 2003 & 274.975 & 3.005 & 52 & 353 \\
\hline 2004 & 70.174 & 3.097 & 8 & 389 \\
\hline 2005 & 147.039 & 3.534 & 37 & 408 \\
\hline 2006 & 258.680 & 4.369 & 78 & 413 \\
\hline 2007 & 496.923 & 3.331 & 148 & 349 \\
\hline 2008 & 632.680 & 3.679 & 259 & 347 \\
\hline 2009 & 406.269 & 3.946 & 174 & 345 \\
\hline 2010 & 1.011 .548 & 3.817 & 300 & 390 \\
\hline 2011 & 764.032 & 4.965 & 191 & 442 \\
\hline 2012 & 589.591 & 3.266 & 121 & 280 \\
\hline 2013 & 1.452 .489 & 4.141 & 235 & 359 \\
\hline 2014 & 589.107 & 4.706 & 60 & 331 \\
\hline 2015 & 1.688 .688 & 4.341 & 140 & 334 \\
\hline 2016 & 1.500 .535 & 2.870 & 176 & 234 \\
\hline Total & 11.100 .213 & 63.779 & 2.144 & 6.093 \\
\hline
\end{tabular}

mos 15 anos foi de $\mathrm{R} \$ 270.739 .122,53$ enquanto a leptospirose teve um custo muito menor, $\mathrm{R} \$ 30.341 .984,22$. Em relação aos custos sociais, é importante salientar que os cálculos de AVP, ATP e perda salarial são geralmente produzidos para anos específicos, mas a literatura científica disponível não apresenta anos coincidentes para ambas as enfermidades analisadas, o que implica compreendermos suas especificidades sem compará-las diretamente.

Souza et al. ${ }^{9}$ identificaram que, em 2007, 6.490 Anos Potenciais de Vida (AVP), 4.617 Anos de Trabalho e R \$22.931.116,00 em salários foram perdidos no Brasil em decorrência da leptospirose, ultrapassando os valores para doenças crônicas de difícil controle como a Aids e a hipertensão no mesmo ano. Os valores para dengue, por sua vez, variam a depender da ocorrência ou não de epidemias no ano, conforme exposto na Tabela 2, mas tendem a superar os valores de doenças como malária, leishmaniose, esquistossomose, hanseníase e infecções meníngeas ${ }^{14}$.

Tendo em vista as limitações comparativas em virtude dos distintos períodos enfocados pelas pesquisas de custos sociais para dengue e leptospirose, gostaríamos de salientar apenas o fato de que ambas superam, em valor e em seus respectivos anos, doenças consideradas problemas de saúde pública, inclusive algumas DTN. Logo, embora os custos hospitalares e sociais, assim como a morbidade e mortalidade, sejam importantes fatores para caracterizar e priorizar um problema de saúde pública, eles não são, em última instância, os únicos determinantes desse processo. Enquanto o número de casos e o custo hospitalar justificariam o investimento na prevenção e tratamento da dengue, o número de óbitos justificaria o investimento em prevenção para a leptospirose. Tendo em vista essa arbitrariedade, nossa hipótese é que a razão dessa atribuição de prioridade à dengue em detrimento da leptospirose encontre-se no perfil da população acometida por cada doença.

\section{Os perfis populacionais da dengue e da leptospirose humana}

Para discutirmos a diferença entre perfis populacionais da leptospirose humana e da dengue, selecionamos na base de dados do Sinan quatro variáveis relacionadas à leptospirose (2007-2015) e à dengue (2007-2012) nos períodos recentes disponíveis na plataforma: idade, sexo, cor/raça e escolaridade. $\mathrm{O}$ total de casos e os valores percentuais foram apresentados na Tabela 3, com destaque aos casos ignorados.

Com relação ao sexo, os homens $(78,6 \%)$ são mais afetados pela leptospirose humana do que as mulheres $(21,3 \%)$. Nos casos de dengue, essa diferença entre casos de homens $(55,1 \%)$ e mulheres $(44,8 \%)$ é bem mais sutil. Com relação ao critério de cor/raça, há predomínio de casos de leptospirose em pessoas que se autodeclaram brancas $(46 \%)$, seguidas de pessoas que se autodeclaram pretas e pardas $(41.2 \%)$, amarelas $(0,5 \%)$ e indígenas $(0,3 \%)$. O percentual de pessoas que se autodeclaram brancas $(28,5 \%)$ nos casos de dengue é inferior ao de pessoas que se autodeclaram pretas e pardas $(35,4 \%)$, sendo ainda menores os casos declarados como amarelos $(0,9 \%)$ e indígenas $(0,3 \%)$.

No quesito escolaridade, a maior parte das pessoas que tiveram leptospirose não chegou a completar a $8^{\text {a }}$ série $(35,6 \%)$. Esse número é bem inferior nos casos de dengue $(18,5 \%)$ e com relação à idade, ambas as enfermidades possuem concentração de casos na extensa faixa etária produtiva que vai dos 20 aos 59 anos, sendo a porcentagem de casos de leptospirose $(72,3 \%)$ maior que a porcentagem dos casos de dengue $(60,6 \%)$. 
Todavia, devemos atentar para o fato de que houve um número elevado de casos ignorados nos quesitos cor/raça e escolaridade para ambas

Tabela 2. Comparativo de custos sociais da dengue em ano epidêmico (2010) e ano não epidêmico (2012).

\begin{tabular}{crrc}
\hline Ano & \multicolumn{1}{c}{ APV } & \multicolumn{1}{c}{ ATP } & Perda salarial \\
\hline 2010 & 13.955 & 8.244 & $\mathrm{R} \$ 56.059 .200,00$ \\
2012 & 7.297 & 5.124 & $\mathrm{R} \$ 42.494 .869,20$ \\
\hline
\end{tabular}

Fonte: Adaptado de Leite ${ }^{14}$. as enfermidades. No caso do quesito cor/raça $12 \%$ dos casos de leptospirose e $34,9 \%$ dos casos de dengue foram ignorados, enquanto no quesito escolaridade $35 \%$ dos casos de leptospirose e 51,56\% dos casos de dengue foram ignorados. Embora possamos caracterizar o perfil populacional de ambas as enfermidades em relação ao sexo e à idade para os respectivos períodos, esse perfil fica incompleto em virtude da impossibilidade de se obter dados precisos sobre cor/raça e escolaridade. Em termos práticos, essa quantidade de casos ignorados indica que, de alguma ma-

Tabela 3. Perfil populacional dos casos de leptospirose (2007-2015) e dengue (2007-2012) no Brasil por sexo, cor/ raça, escolaridade e idade.

\begin{tabular}{|c|c|c|c|c|c|}
\hline & \multirow{2}{*}{ Dados } & \multicolumn{2}{|c|}{ Número Absolutos* } & \multicolumn{2}{|c|}{ Valores Percentuais ${ }^{\star}$} \\
\hline & & Dengue & Leptospirose & Dengue & Leptospirose \\
\hline \multirow[t]{3}{*}{ Sexo } & Mulheres & 1.671 .459 & 7.367 & 44,8 & 21,3 \\
\hline & Homens & 2.057 .939 & 27.162 & 55,1 & 78,6 \\
\hline & Ignorados & 1.134 & 3 & $<0,1$ & $<0,1$ \\
\hline \multirow[t]{6}{*}{ Cor/Raça } & Brancos & 1.064 .733 & 15.874 & 28,5 & 46 \\
\hline & Pretos & 161.060 & 1.873 & 4,3 & 5,5 \\
\hline & Amarelos & 34.704 & 205 & 0,9 & 0,5 \\
\hline & Pardos & 1.153 .495 & 12.329 & 30,9 & 35,7 \\
\hline & Indígenas & 14.057 & 99 & 0,3 & 0,3 \\
\hline & Ignorados & 1.302 .483 & 4.152 & 34,9 & 12,0 \\
\hline \multirow[t]{11}{*}{ Escolaridade } & Analfabetos & 33.730 & 562 & $<0,1$ & 1,6 \\
\hline & $1^{\text {a }}$ a $4^{\text {a }}$ serie incompleta & 219.056 & 3.597 & 5,8 & 10,5 \\
\hline & 4 série completa & 135.522 & 2.226 & 3,6 & 6,5 \\
\hline & $5^{\mathrm{a}}$ a $8^{\mathrm{a}}$ série incompleta & 333.571 & 5.902 & 9,0 & 17,0 \\
\hline & Ensino fundamental completo & 167.398 & 2.474 & 4,4 & 7,2 \\
\hline & Ensino médio incompleto & 203.096 & 2.344 & 5,4 & 6,8 \\
\hline & Ensino médio completo & 294.539 & 3.616 & 7,8 & 10,5 \\
\hline & Ensino superior incompleto & 50.312 & 441 & 1,3 & 1,2 \\
\hline & Ensino superior completo & 80.943 & 584 & 2,6 & 1,7 \\
\hline & Não se aplica & 288.639 & 678 & 7,7 & 2,0 \\
\hline & Ignorados & 1.923 .726 & 12.108 & 51,56 & 35,0 \\
\hline \multirow[t]{12}{*}{ Idade } & $<1$ ano & 58.672 & 243 & 1,5 & 0,7 \\
\hline & $1-4$ & 134.427 & 182 & 3,6 & 0,5 \\
\hline & $5-9$ & 252.335 & 880 & 6,7 & 2,5 \\
\hline & $10-14$ & 355.456 & 2.177 & 9,5 & 6,5 \\
\hline & $15-19$ & 388.576 & 3.355 & 10,4 & 9,7 \\
\hline & $20-39$ & 1.434 .648 & 14.188 & 38,4 & 41,0 \\
\hline & $40-59$ & 828.264 & 10.758 & 22,2 & 31,3 \\
\hline & $60-64$ & 101.990 & 1.210 & 2,7 & 3,5 \\
\hline & $65-69$ & 71.133 & 732 & 1,9 & 2,1 \\
\hline & $70-79$ & 77.112 & 674 & 2,0 & 1,9 \\
\hline & $80 \mathrm{e}+$ & 22.614 & 122 & 0,6 & 0,3 \\
\hline & Ignorados & 5.288 & 11 & 0,1 & $>0,1$ \\
\hline
\end{tabular}


neira, as informações referentes a esses quesitos nem sempre são reportadas no formulário correspondente ou, no caso de serem corretamente preenchidas, não são encaminhadas e registradas apropriadamente no sistema. Logo, essa informação torna-se invisível.

Quando há uma lacuna nas informações, outro curso de ação pode ter lugar: ignorar algo é tomar uma atitude em relação ao que está sendo ignorado, excluindo-o dos espaços de discussão. Parece que não é possível definir as características básicas da população que teve leptospirose e dengue por meio dos dados epidemiológicos porque não há informações suficientes. Desse modo essas questões não corroboram para delimitar o campo de ações de saúde voltadas à prevenção e tratamento de ambas as enfermidades. Afinal, como agir sem saber esses dados básicos sobre a população? O fato é que se sabe muito bem a diferença populacional entre a leptospirose e a dengue, mas essa diferença não está dada nos números, mas na ação de seus vetores e seus determinantes sociais.

A dengue é transmitida por uma espécie de mosquito chamada Aedes Aegypti que mede aproximadamente $0,5 \mathrm{~cm}$ de comprimento. A fêmea dessa espécie precisa da proteína do sangue para promover a maturação de seus ovários e o desenvolvimento de seus ovos e, portanto, busca nutrição sob a pele de outras espécies. Diferentemente de outros mosquitos, as fêmeas de Aedes Aegypti podem picar mais de uma pessoa durante esse período e, não necessariamente, no mesmo ambiente. Seu alcance de voo é de 300 metros, mas a fêmea em período de reprodução pode voar até três quilômetros em busca de um local adequado para desova. Esse local pode ser qualquer espaço com água, de preferência, parada e cristalina.

O principal vetor da leptospirose humana, por sua vez, é o rato, com destaque para a espécie Rattus Norvegicus, originária da Ásia, mas atualmente difundida por todas as regiões habitadas do planeta. A espécie se expandiu do Leste Asiático substituindo as populações de Rattus Rattus em virtude de seu tamanho (em média $25 \mathrm{~cm}$ e $300 \mathrm{~g}$ ), resistência, agressividade e capacidade de produzir buracos e habitar locais de difícil acesso próximos a habitações humanas. Atualmente, a espécie do Rattus Norvegicus vive em condição de sinantropismo, buscando espaços construídos por humanos nos quais haja disponibilidade de água e alimentos constantes. Ele tem hábitos noturnos e circula por um território específico, geralmente próximo de onde estabeleceu seu ninho ${ }^{15}$.
A diferença entre essas espécies como vetores de doenças é sua relação com os seres humanos e seu espaço de circulação. Para que o vírus da dengue seja transmitido, os mosquitos devem voar de encontro aos seres humanos, entrando em contato direto com nosso corpo. Com relação à leptospirose é necessário estar em um local em que alimentos e dejetos estejam em condições de serem alcançados pelos ratos infectados, que os ratos urinem em um local acessível a seres humanos, que os seres humanos entrem em contato com a urina do rato infectado e que a bactéria consiga entrar na corrente sanguínea.

$\mathrm{Na}$ batalha para provar a melhor eficácia vetorial, o Rattus Norvegicus perde porque seu interesse alimentar não está no corpo humano e porque sua circulação é restrita por seus hábitos e sua anatomia: ele é incapaz de alçar voo. Os mosquitos são vetores muito mais eficientes, alcançando grupos populacionais mais abrangentes e transmitindo com mais eficácia o vírus da dengue. Por esse motivo, a população afetada pela dengue é mais ampla e heterogênea do que aquela afetada pela leptospirose (a distribuição sexual e por faixa etária apresentada na Tabela 3 indica essa heterogeneidade do perfil populacional da dengue). O Aedes Aegypti é mais democrático na distribuição do vírus.

E qual seria então a população afetada pela leptospirose humana? Para chegarmos a essa conclusão é necessário associarmos as características de seu principal vetor aos determinantes sociais da doença. Os determinantes sociais são um conjunto de critérios econômicos, ambientais, culturais e psicológicos que caracterizam a condição de vida e trabalho das pessoas e grupos populacionais e determinam sua situação de saúde. Esses determinantes, conhecidos pela sigla DSS, têm sido particularmente importantes para compreender as iniquidades em saúde; desigualdades entre grupos populacionais que além de sistemáticas e relevantes são evitáveis ${ }^{16}$. Fatores comportamentais como a eliminação de água parada em espaços propícios à reprodução do mosquito no caso da dengue, ou questões de infraestrutura como saneamento básico no caso da leptospirose humana, não apenas indicam características de uma enfermidade como apontam, sobretudo, para o modo pelo qual diferentes enfermidades adentram corpos diferentes.

As pesquisas da área apontam que as pessoas que são infectadas e desenvolvem a leptospirose geralmente moram em áreas sem saneamento como favelas e assentamentos precários e/ou trabalham em locais ou exercem atividades nas 
quais o contato com a urina do rato é possível ${ }^{17,18}$. Essas pessoas são marcadas pela pobreza, segregação racial e baixa escolaridade, justamente os dados mais subnotificados. Por isso, a invisibilidade dos dados mantém a população em situação de vulnerabilidade à leptospirose marginalizada em relação aos cuidados com a doença. Essa segregação populacional define o que Foucault ${ }^{19}$ denominou de Racismo de Estado:

Com efeito, que é o Racismo? É, primeiro, o meio de introduzir afinal, nesse domínio da vida de que o poder se incumbiu, um corte: o corte entre o que deve viver e o que deve morrer. No contínuo biológico da espécie humana, o aparecimento das raças, a distinção das raças, a hierarquia das raças, a qualificação de certas raças como boas e de outras, ao contrário, como inferiores, tudo isso vai ser uma maneira de fragmentar esse campo do biológico de que o poder se incumbiu; uma maneira de defasar, no interior da população, uns grupos em relação a $\operatorname{outros}^{19}$ (p.214).

$\mathrm{Na}$ ampliação do poder soberano para o poder sobre a vida, o direito soberano de deixar viver e fazer morrer é invertido para um direito de fazer viver aqueles a quem interessa o Estado e deixar morrer aos "inferiores". Portanto, como nos aponta Foucault19, "o racismo é ligado ao funcionamento de um Estado que é obrigado a utilizar a raça, a eliminação das raças e a purificação da raça para exercer seu poder soberano"19(p.217). A ênfase estatal na dengue se apresenta nesses termos porque a população com melhores condições sociais para o enfrentamento à doença também pode adoecer, sendo essa uma população muito mais valiosa na perspectiva do Estado do que a população em situação de vulnerabilidade à leptospirose.

Se fossemos considerar os dados previamente apresentados, dificilmente chegaríamos a essa conclusão. Eis uma invisibilidade da leptospirose que a torna uma doença negligenciada: ao contrário da dengue, a falta de dados nos casos de leptospirose oblitera uma população muito específica, historicamente negligenciada por outros mecanismos de governo. Logo, compreende-se que do ponto de vista do perfil populacional a política pública de saúde opta por investir em uma doença que pode afetar de alguma forma as elites do país do que uma doença que afeta um grupo social específico: pobre, negro, periférico e tornado invisível para a política pública de saúde.

Mas este não é o único ponto cego que produz a dupla negligência em relação à leptospirose. Não apenas o perfil populacional dessas doenças varia como também seu quadro clínico.
Às vezes, a leptospirose é rapidamente diagnosticada. Outras vezes, não é possível ver sequer sua manifestação e, eventualmente, ela é confundida com a dengue. Logo, não são apenas os números que obliteram. A resposta do corpo às infecções também interfere no processo de tornar a doença visível.

\section{O caráter mimético da leptospirose humana}

Mesmo tendo em vista que os perfis populacionais da dengue e da leptospirose são de fundamental importância para a caracterização dessas doenças, sua prevenção e tratamento, nem sempre eles são considerados necessários no processo de definir e diferenciar uma doença da outra. Os diagnósticos de casos, por exemplo, tendem a privilegiar o critério clínico-laboratorial sobre o critério clínico-epidemiológico, no qual esse perfil populacional se expressa. Isso ocorre porque os dados laboratoriais podem expressar relações que os olhos e o tato das médicas e dos médicos não podem acessar para realizar um diagnóstico. Além disso, os dados epidemiológicos não são totalmente confiáveis: como vimos na parte anterior, eles podem ser inexistentes, inespecíficos ou imprecisos. Tudo o que os profissionais de saúde e os usuários não querem enquanto estão em busca de soluções para os problemas que afligem pessoas doentes são esses qualificadores.

Apesar dos critérios diagnósticos serem distintos, a presença dos sinais e sintomas é um fator crucial para ambos os casos. Mas nem sempre eles estão presentes. A manifestação da leptospirose nos corpos de pessoas específicas também pode gerar invisibilidade. Em alguns casos, os sinais e sintomas são claros e o diagnóstico preciso antes mesmo do exame, enquanto em outros, a leptospirose apenas existe em exames específicos, sendo clinicamente invisível. O trecho abaixo é de um diário de campo produzido em julho de 2015 que ilustra um caso clinicamente visível da doença:

Ao entrarmos na sala ficamos impressionados com o que vimos. Era uma mulher, com aproximadamente 50 anos, deitada em uma maca com a pele completamente alaranjada, gemendo de dor com os olhos fechados. Ao seu lado, uma jovem com o olhar calmo que dizia baixinho "Calma, mãe. Os médicos vão resolver". E olhou para nós dois, eu e a estudante de enfermagem, como quem pede uma resposta confirmatória. Lembro-me quando a médica pediu para que a usuária abrisse rapidamente os olhos. Seus olhos negros estavam saltados, com as escleras (parte branca do olho) alaranjadas e veias 
oculares pronunciadas como teias vermelhas. Ela os fechou rapidamente. A luz hospitalar que incidia sobre os olhos a maltratava. No prontuário, a médica escrevera: febre, congestão conjuntival, icterícia, mialgia, dor na panturrilha, insuficiência respiratória, cefaleia, vômito, prostração, diarreia, dor abdominal e colúria (urina escura). Todos os sintomas típicos de leptospirose. Não faltava nenhum. "Você já tinha visto um caso assim?" - perguntou a médica dirigindo-se a mim. "Não, não... nunca vi um caso assim". Eu acompanhei a mulher e sua filha por algumas semanas. Ela foi encaminhada para um hospital referência em hemodiálise e fui alguns dias visitá-las. Na última vez que as vi, a situação havia piorado. Na sala da UTI, a usuária estava balbuciando, sem conseguir se comunicar, com os lábios completamente secos e grudados. Ela faleceu poucos dias depois.

O caso dessa usuária é prototípico. Os sinais e sintomas, os exames laboratoriais, bem como os antecedentes epidemiológicos eram totalmente congruentes com o diagnóstico de caso para leptospirose humana. A médica identificou o caso clinicamente. Essa é uma versão da leptospirose que se manifesta de forma visível no corpo.

Nem sempre, todavia, a leptospirose se manifesta de maneira tão evidente e tão grave. Às vezes, o corpo não expõe aquilo que precisamos ver. Ele também esconde. Foi o que aconteceu a um usuário que teve leptospirose, mas a doença não foi identificada nem por critérios epidemiológicos, nem clínicos ou laboratoriais simples, não específicos para a enfermidade. $O$ trecho abaixo é de um diário de campo produzido em julho de 2015 e relata o momento em que uma médica me apresenta um usuário que foi infectado enquanto trabalhava em uma obra de construção civil. Todavia, a doença não se manifestou da maneira esperada:

A médica contou que ele havia sido diagnosticado alguns dias antes com dengue, mas no retorno para avaliação, outra médica solicitou exames para leptospirose em virtude de ele ter relatado contato com água suja em seu local de trabalho. Os exames confirmaram a infecção. O usuário teve as duas enfermidades ao mesmo tempo. Fiquei um pouco assustado. Não sabia que isso era possível. A primeira coisa que me veio à cabeça foi perguntar se ele estava bem. O próprio usuário respondeu desconcertado que sim, com umas dores de cabeça, mas nada mais. Depois a médica me explicou que o usuário teve uma forma branda de ambas as enfermidades na qual predominou a dengue.

A leptospirose é conhecida por ser uma doença mimética: sua versão mais branda tende a ser confundida com outras doenças como a dengue, o que tem exigido um aprimoramento das práticas de monitoramento e diagnóstico com vistas à ampliação da detecção dos $\operatorname{casos}^{20}$. Essa confusão diagnóstica tem despertado a atenção dos estudiosos da área porque as infecções por diferentes patógenos que exibem sintomas similares requerem tratamentos diferentes, sendo a aplicação de um tratamento incorreto um fator que eleva as chances de óbito ${ }^{21}$. Atualmente, a dor retro-orbital (atrás dos olhos) é utilizada pelos médicos como sintoma de referência para o diagnóstico diferencial da dengue enquanto a dor na panturrilha é um sintoma para o diagnóstico diferencial da leptospirose.

Todavia, nem sempre as coisas saem conforme os livros de medicina. Às vezes o que se faz invisível não são números, mas sinais e sintomas. Se não há sinais e sintomas, não há o que ser relatado. Se não há o que ser relatado, não há diagnóstico e o problema não existe, ao menos formalmente. O usuário não seria representado por um número nos quadros de leptospirose que mostramos anteriormente. Ele seria apenas mais um número para a dengue.

Isso chama a atenção para a questão da subnotificação de casos de leptospirose. Quantos casos da doença são diagnosticados como dengue na rede de saúde e o quanto isso contribui para a valorização de uma enfermidade em detrimento de outra? Estudos sobre a subnotificação de casos de leptospirose no Brasil têm apontado essa fragilidade. Em Fortaleza, considerando os casos reportados pelas autoridades de vigilância como dengue que foram posteriormente descartados com base em testes de laboratório, estima-se que o número de casos de leptospirose possa ser 26 a 49 vezes maior que o diagnosticado e reportado pelos serviços de saúde ${ }^{22}$.

Além disso, há trabalhos que chamam a atenção para o fato de que o aumento no número de registros de casos de leptospirose em período chuvoso pode decorrer de uma maior atenção e ênfase dos profissionais de saúde em identificar sintomas da doença nesse período, sendo as associações e correlações coincidentes ou mesmo acidentais em muitos casos, enquanto nos períodos não chuvosos essa atenção não é requerida ${ }^{23}$.

Por fim, no caso desse usuário, tanto a dengue como a leptospirose foram brandas, de modo que seu próprio corpo conseguiu resistir às enfermidades. É um caso no qual a solicitação da sorologia foi fundamental para que ele não fosse excluído das estatísticas. Logo, questionar essa invisibilidade clínica implica decisões sobre os locais nos quais se realizarão intervenções e os 
corpos que serão afetados por elas: tendo em vista que os corpos reagem de maneiras distintas à leptospirose, um colega de trabalho deste usuário, nas mesmas condições, talvez não tivesse desenvolvido apenas uma forma branda da doença.

\section{Conclusão}

Como a definição de um problema de saúde pública é sempre uma disputa, é necessário justificar a nossa classificação da leptospirose humana nesses termos. A nossa escolha por estudar essa enfermidade decorre de um conjunto de fatores que podem ser resumidos da seguinte maneira: ela é uma doença de alto impacto, porém duplamente negligenciada no setor de saúde em virtude de critérios pouco claros para elencar prioridades e da produção de invisibilidades relativas à população por ela afetada e ao seu quadro clínico.

Para chegar a essa conclusão mostramos como os critérios de morbidade, mortalidade e custos hospitalares e sociais são ambíguos para justificar uma tomada de decisão que classifique determinada enfermidade como problema de saúde pública que precisa de investimento maciço em detrimento de outra. Além disso, buscamos mostrar que o perfil populacional da doença e de sua manifestação clínica pode gerar invisibilidades que contribuem para que a leptospirose humana seja menos importante do que a dengue no espaço público. Ao dar visibilidade aos determinantes sociais da doença e aos deslizes diagnósticos em relação a ela, chamamos a atenção para o fato de que a leptospirose humana acomete uma população que o Estado não tem interesse em manter viva enquanto a dengue acomete um grupo mais amplo de pessoas, inclusive aquelas que o Estado tem o interesse de preservar.

Logo, o que se passa com a leptospirose é que ela é uma doença da pobreza, com uma população camuflada pela invisibilidade de dados populacionais e cujo mimetismo e sazonalidade geram invisibilidades clínico-diagnósticas que impedem um reconhecimento maior da população acometida pela doença. Logo, ela se torna um alvo fácil do racismo de Estado, sendo a visibilidade dessas questões a primeira forma de enfrentamento a essa mecânica que não apenas negligencia duplamente a doença, mas as pessoas por ela afetadas.

\section{Colaboradores}

MHM Martins participou da concepção, desenho, análise e interpretação dos dados e também da redação do artigo. MJP Spink participou da revisão crítica e aprovação da versão a ser publicada.

\section{Agradecimentos}

Os autores agradecem ao CNPq pela bolsa de doutorado concedida e aos integrantes do grupo Laicos da Universidade Autônoma de Barcelona pelas discussões que fomentaram a produção desse artigo. 


\section{Referências}

1. Ko AI, Goarant C, Picardeau M. Leptospira: The Dawn of the Molecular Genetics Era for an Emerging Zoonotic Pathogen. Nat Rev Microbiol 2009; 7(10):736-747.

2. Haake DA, Levett, PN. Leptospirosis in Humans. Curr Top Microbiol Immunol 2015; 387:65-97.

3. Ko AI, Reis M, Dourado M., Johnson-Júnior W, Riley L. Urban epidemic of severe leptospirosis in Brazil. Lancet 1999; 354(9181):820-825.

4. Hotez PJ. Global urbanization and the neglected tropical diseases. PLoS Negl Trop Dis 2017; 11(2):e0005308.

5. Araújo WN, Finkmoore B, Ribeiro GS, Reis RB, Felzemburgh, RD, Hagan JE, Reis MG, Ko AI, Costa F. Knowledge, Attitudes, and Practices Related to Leptospirosis among Urban Slum Residents in Brazil. Am J Trop Med Hyg 2013; 88(2):359-363.

6. Guimarães R, Cruz O, Parreira V, Mazoto M, Vieira J, Asmus, C. Análise temporal da relação entre leptospirose e ocorrência de inundações por chuvas no município do Rio de Janeiro, Brasil, 2007-2012. Cien Saude Colet 2014; 19(9):3683-3692.

7. Hotez PJ. Forgotten people, forgotten diseases: the neglected tropical diseases and their impact on global health and development. Washington: ASM Press; 2008.

8. Hotez PJ, Fujiwara R. Brazil's neglected tropical diseases: an overview and a report card. Microbes Infect 2014; 16(8):601-606.

9. Souza V, Arsky M, Castro A, Araujo W. Anos potenciais de vida perdidos e custos hospitalares da leptospirose no Brasil. Rev Saude Publica 2011; 45(6):10011008.

10. Cavaca A, Vasconcellos-Silva P. Doenças midiaticamente negligenciadas: uma aproximação teórica. Interface (Botucatu) 2015; 19(52):83-94.

11. Halliday JE, Allan KJ, Ekwem D, Cleaveland S, Kazwala RR, Crump JA. Endemic zoonoses in the tropics: a public health problem hiding in plain sight. Vet Rec 2015; 176(9):220-225.

12. Brasil. Ministério da Saúde (MS). Doenças negligenciadas: estratégias do Ministério da Saúde. Rev Saude Publica 2010; 44(1):200-202.

13. Galindo D, Martins M, Rodrigues R. Jogos de armar: narrativas como modo de articulação de múltiplas fontes no cotidiano de pesquisa. In: Spink MJ, Brigagão J, Nascimento V, Cordeiro M, organizadoras. A produção de informação na pesquisa social: compartilhando ferramentas. Rio de Janeiro: Centro Edelstein de Investigações sociais; 2014. p. 295-323.

14. Leite P. Impacto da dengue no Brasil em período epidêmico e não epidêmico: incidência, mortalidade, custo hospitalar e Disability Adjusted Life Years (DALY) [dissertação]. Brasília: Universidade de Brasília; 2015.

15. Santoianni F. Todos os ratos do mundo: do flautista de Hamelin a Mickey Mouse: o irresistivel charme dos roedores. São Paulo: Best Seller; 1993.
16. Buss P, Pellegrini-Filho A. A saúde e seus determinantes sociais. Physis 2007; 17(1):77-93.

17. Hagan JE, Moraga P, Costa F, Capian N, Ribeiro S, Wunder E A, Felzemburgh RD, Reis RB, Nery N, Santana FS, Fraga D, Santos BL, Santos AC, Queiroz A, Tassinari W, Carvalho MS, Reis MG, Diggle PJ, Ko AI. Spatiotemporal Determinants of Urban Leptospirosis Transmission: Four-Year Prospective Cohort Study of Slum Residents in Brazil. PLoS Negl Trop Dis 2016; 10(1):e0004275.

18. Gonçalves N, Araújo E, Sousa Júnior A, Pereira W, Miranda C, Campos P, Matos M, Palácios V. Distribuição espaço-temporal da leptospirose e fatores de risco em Belém, Pará, Brasil. Cien Saude Colet 2016; 21(12):3947-3955.

19. Foucault M. Em defesa da sociedade. WMF Martins Fontes: São Paulo; 2010.

20. Izurieta R, Galwankar S, Clem A. Leptospirosis: The "mysterious" mimic. J Emerg Trauma Shock 2008; 1(1):21-33.

21. Priya SP, Sakinah S, Sharmilah K, Hamat RA, Sekawi Z, Higuchi A, Ling MP, Nordin SA, Benelli G, Kumar SS. Leptospirosis: molecular trial path and immunopathogenesis correlated with dengue, malaria and mimetic hemorrhagic infections. Acta Trop 2017; 176(1):206-223.

22. Fontes RM, Cavalcanti LPG, Oliveira ACA, Bezerra LFM, Gomes AMM, Colares JKB, Lima DM. A new possibility for surveillance: do we identify all cases of leptospirosis? Rev Inst Med Tropical São Paulo 2015; 57(5):443-446.

23. Àvila-Pires FD. Leptospirose e enchentes: uma falsa correlação? Rev Patologia Tropical 2006; 35(3):199204.

Artigo apresentado em 31/03/2018

Aprovado em 25/06/2018

Versão final apresentada em 27/06/2018 